Al-Fusha: Arabic Language Education Journal

Vol. 1 No. 2 Juli 2019 | Hal. 37-44

ISSN (Cetak): 2655-6162 | ISSN (Online): 2655-8025

https://ejournal.inaifas.ac.id/index.php/alfusha/article/view/347

\title{
اللغة العربية في سياق اللغات السـامية
}

\section{Bahauddin}

STAI Taswirul Afkar Surabaya

(bahauddin90@gmail.com)

\begin{abstract}
The birth of Arabic in the form of what is now started from a long civilization in the past. The scientists then classified the language, including the language revealed by Noah>s children who later spread in various languages in the world, especially Arabic. Sam language became the language of his Arabic ancestors. But what is the difference between these languages and other European languages? This needs to be known so that the horizon related to the language is wide open.
\end{abstract}

Keywords: arabic (العربية), Sam (سام), language(اللغه)

\section{تمهييد}

(اللغات السامياة) مصطلح حديث يطلق على مجموعة من اللغات المتقاربة نسبة إلى سام

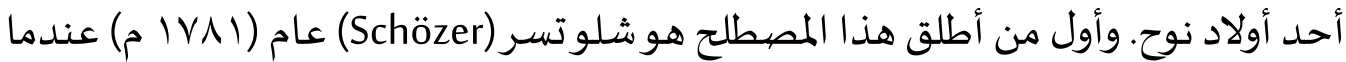
كان يبحث عن تسمية مشتركة للعبر بين والعرب والأحباش الذين ظهر فى لغاتهم نشاباه

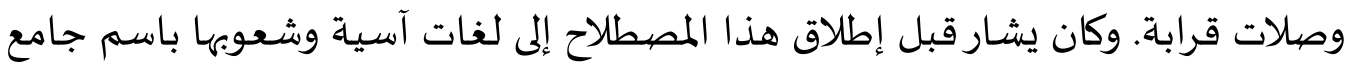
اللغات والشعوب الشرقياة.

فالساميون هم أبناء سام بحسب ما ورد في النص التوراتي الذي أتى على ذكرشعوبهم وفق

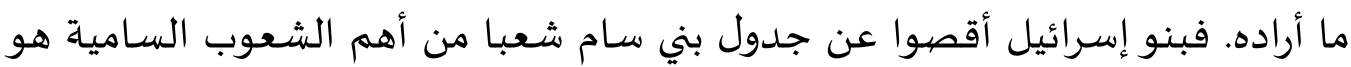


الشعب الكنعاني، وأدخلوا بالمقابل شعيب آخرين هما (عيلام) و (لود) فى جدول بني سام،

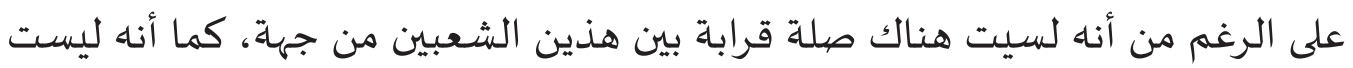
بينهما وبين الشعوب السامية أي من جهة أخرى.

ومع أن كلمة (سـامية) غذت علما على فرع من الدراسات اللغوية في هذا العصر، فإن بعض

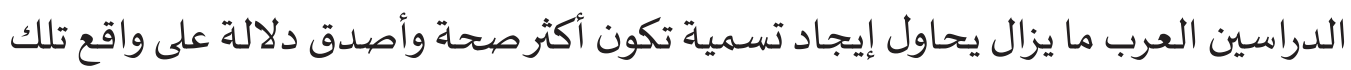

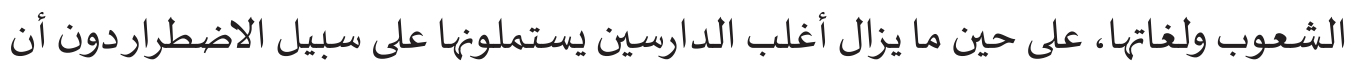

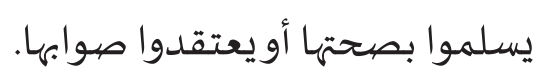

وهناك آراء لايستهان بها في هذا الصدد، فقد ضاق بكلمة (سامية) الكثير من علمائنا

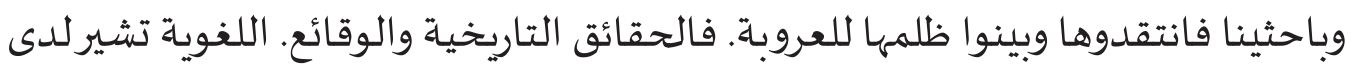
هؤلاء الباحثين إلى أن (العروبة) حقيقة تاريخية ثابتة تدل على موطن واحد محددد سكنتـه

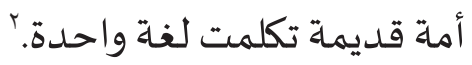

لايستطيع المؤرخون أن يجزموا براء قاطع أو يأخذوا بدليل علمي يبينون به الأصل اللغوي

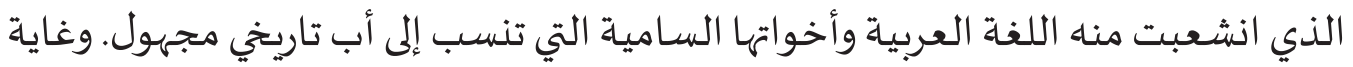
ماتوصل إليه الباحثون أن اللغة العربية هي إحدى اللغات التي أطلق عليها العالم شلوتزر Schözer النبي نوح المذكور في التوراة.

ومن هذه اللغات السامية: البابلية - الآشورية ، والعبرية ، والآرامية (ومنها السريانية) ، والكنعانية ، والجبشية ، وقد انقرض جل هذه اللغات.

ويختلف المؤرخون في موطن اللغات السامية الأول اختلافههم في موطن الأمم السامية الأول، ويميل جمهورهم إلى أن مهد هذه الأمم كان في أرض بابل ، في العراق ، ومن ثم انتشروا في شتى الأنحاء.

وكذلك يختلفون فى أي هذه اللغات أقرب إلى السامية الأم ، ويرى بعض المتشرقين أنها

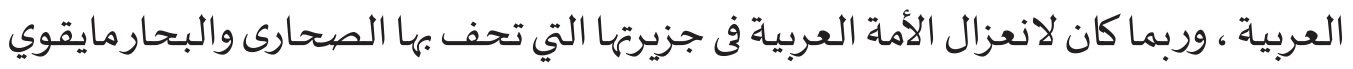

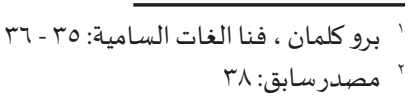


هذا الرأي ، ولاسيما أن علماء اللغات قد وجدوا في بهذا الرأي لعدم توافر الأدلة. ومههما

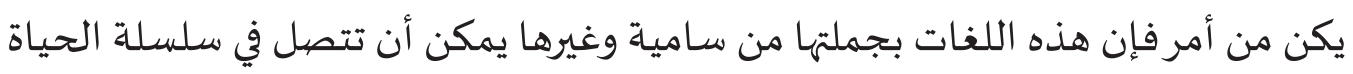

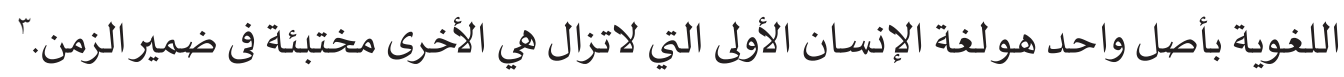

أقسام اللغات السـامية (العربية)

تقسم اللغات السامية إلى شرقية وغربية ، كما تقسم الغربية إلى غربية شمالية وغربية جنوبية.

ا. فالسـامية الشرقية هي الأكادية بفرعيها البابلية والآشـورية ، ونقوشها مكتوبة بالخط

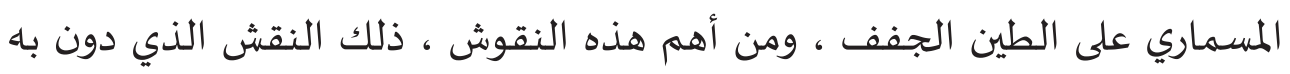

$$
\text { قانون (حمرابي) المشهور. }
$$

والأكادياة تنسب إلى مدينة (أكاد) التي بناها سرجون في الجزء الشمالي من أرض بابل حوالي

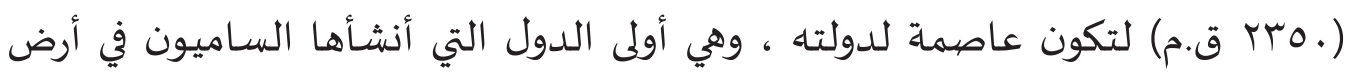

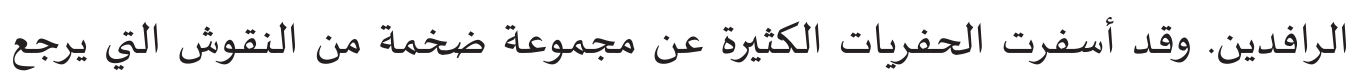

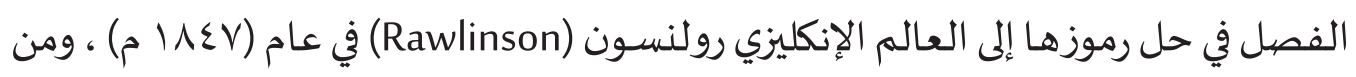
الجدير بالذكر همنا أن سكان تلك المنطقة قبل أن يهاجر إليها الساميون هم شعيب مجههول

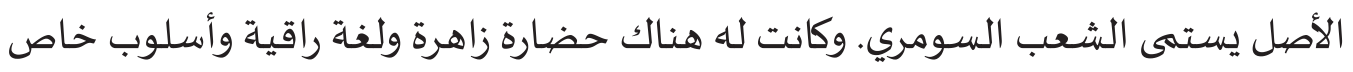
في الرسم عرف بالخط المسماري.

r. أما السامية الغربية فتقسم إلى قسمين : سامية غربية شمالية ، وسامية غربية جنوبية. أ. فالسامية الغربية الشمالية تضم الكنعانية والآرامية

وتقسم الكنعانية إلى شمالية وجنوبياة، والكنعانية لغة تلك الشعوب السامية التى تزحت

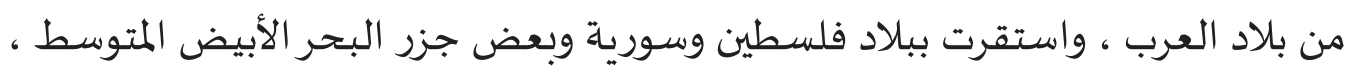

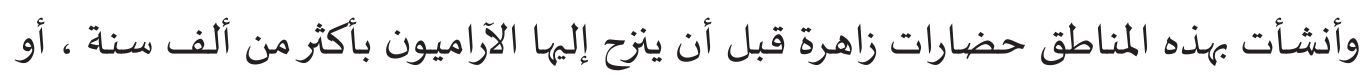

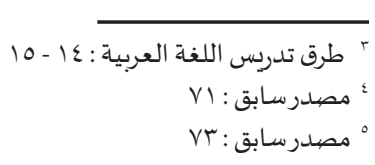


وتمثل اللغة (الأوغاريتية) المنسوبة إلى أوغاريت الشمالي من الكنعانية، فقد عثرعام (9 9 ( )

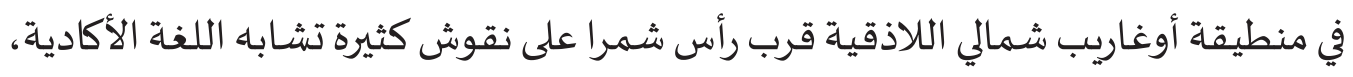

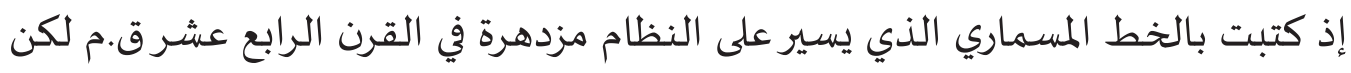

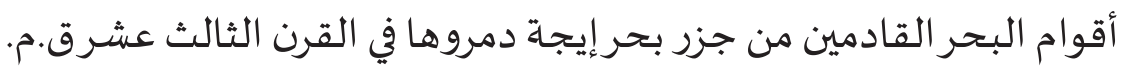

أما الكنعانية الجنوبية فتمثل في اللغة العربية التي كنت بها (العهد القديم) ومن أقدم

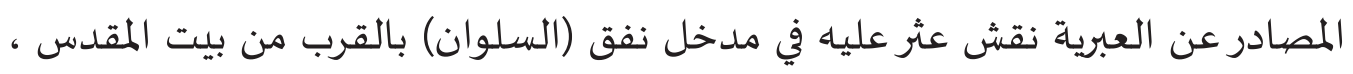

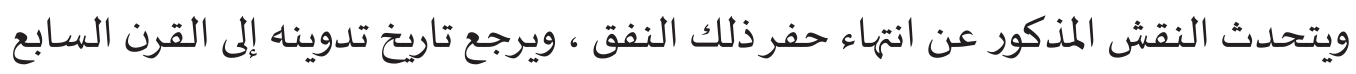

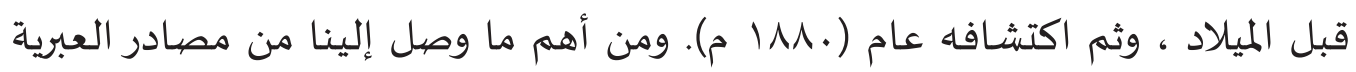

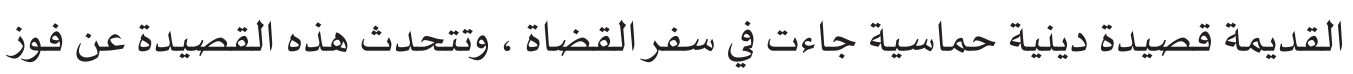

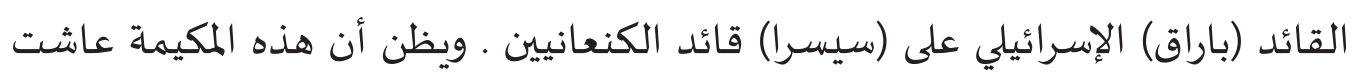
حوالي القرن الثاني عشرقبل الميلاد.

ويضم القسم الثاني من أقسام السـامية الغربية الشمالية (اللغة الآرامية) والآرامية لغة

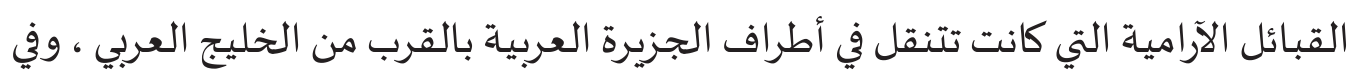

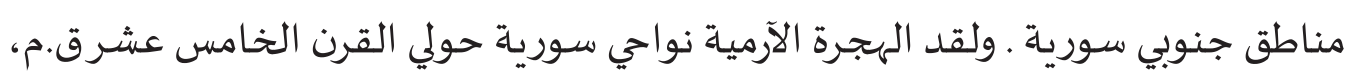

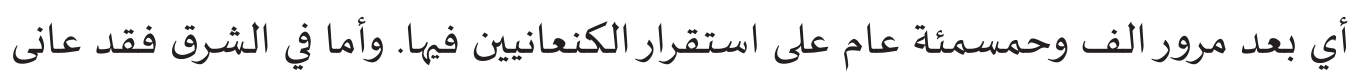

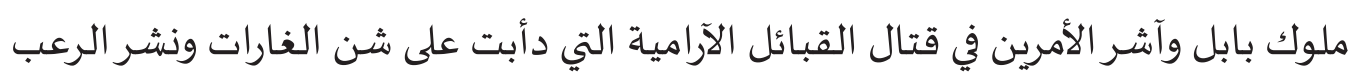

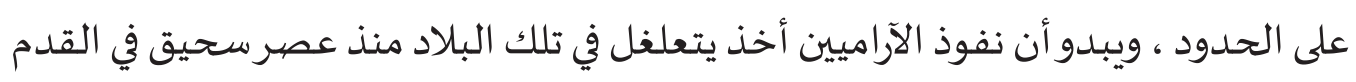

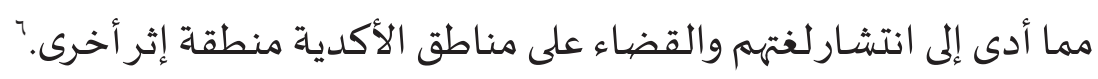

كما اشتبكت الآرامية مع لغات الكنعانيين ، فقضت على العربية وعلى الفينيقية وأصبحت

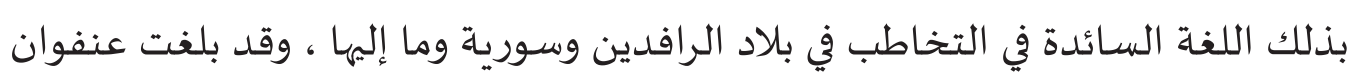

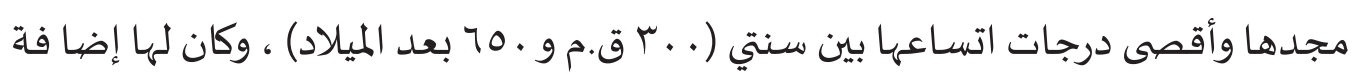

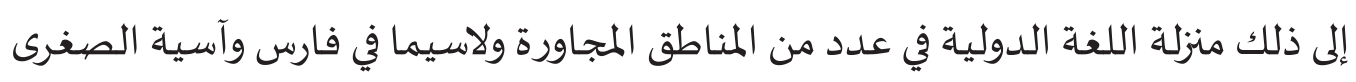

ومصر

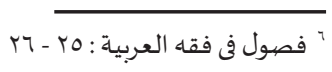




\section{أشهر الآراء في فصيائل اللغات}

وقد اختلف العلماء اختلافا كبيرا فى تقسيم اللغات تهها لاختلافهم في الأسساس الذي يقوم علياه هذا التقسم.

ولكن أشهر النظريات وأميلها بهذا الصدد هي نظرية مكس مولر Max Muller التى تراعى فى

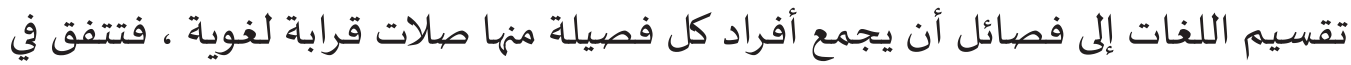
أصول الكلمات وقواعد البنية وتركيب الجمل ..... وما إلى ذلك ، وتكون من الأمم الناطقة بها

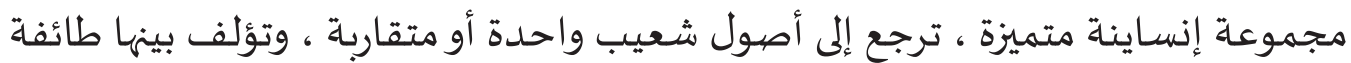

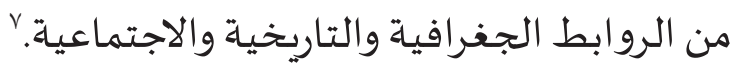

وعلى هذه الأسس ترجع نظرية مكس نظري مكس مولر جميع اللغات الإنسانية إلى ثلاث

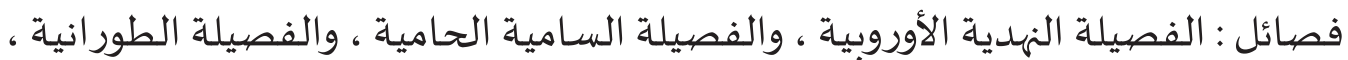
وسنتكلم على كل فصيلة منها على حدة فما يلى:

الفصيلة الأولى : الهندية الأوروبية Langues Indo - Européennes

$$
\begin{aligned}
& \text { تشمل هذه الفصيلة ثمان طوائف من اللغات ، وهي : } \\
& \text { ا. (( اللغات الهندية - الإيرانية )) أو (( اللغات الآرية )) وتشلم شعبتين : }
\end{aligned}
$$

إحداهما شعبة اللغات الهندية (السنسكريتية Sanskrit والبراكريتية Parkrit واللغات

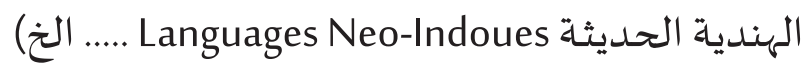
والأخرى شعبة اللغات الإبرانية (الفارسية القديمة Vie perse ؛ والأفستية والزند - أفستية

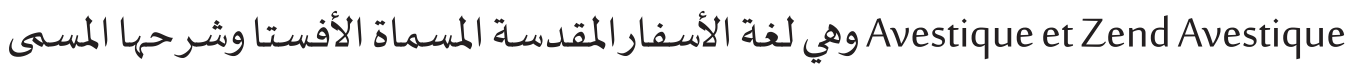
الزند - أفستا ؛ والبهلوية Pehlvi ؛ والفارسية الحديثة Neo - Persan ؛ والكردية Kurde؛

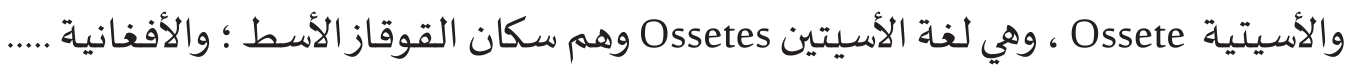


r. ( ( اللغات الإغريقية )) (وتشمل اللغات اليونانية القديمة ، وأشهرهذه اللغات : اليونية - الاتيكية ، والدورياة ؛ وتشمل كذلك اللغات اليوناية التى تكون في القرون السـابقة للميلاد وقادمت على أنقاض اللغات اليونانية القديمة ، واشهرت عند علماء اللغات باسم ((اليوناينة الحديثة)) ؛ وتشمل كذلك اللغات اليونانية في العصر الحاضر).

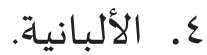

O. Ombrien - Osque و والأمبرية السمنية اللغات الإيطالية" (وتشمل الأسكية

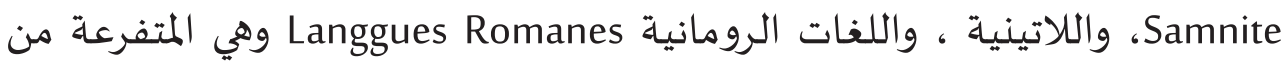
اللاتينية كالفرنيسة والبرتغالية والإيطالية والإسبانية ولغة رومانيا ..... الخ).

$$
\begin{aligned}
& \text { 7. "اللغات الكلتية" Langues Celtiques (التي كانت) } \\
& \text { VLanguages Germaniques" ل "اللغات الجرمانية } \\
& \text { ᄉ. "اللغات البطيقية - السلافية"وتشمل شعبتين : }
\end{aligned}
$$

إحدهما : شعباة اللغات البلطيقية : وهي الليتوانية Lituanienne (لغة لتوانيا Lettonie) واللتونية Lette (لغة ليتونيا Lituanie أو لاتفيا Latvia) والبروسية القديمة: والأخري شعبة اللغات السلافية أو الصقلبية :وهي السلافية

\section{الفصيلة الثانية : الحامية - السامية Langues Chamito - Semitques}

تشمل هذه الفصيلة مجموعتين من اللغات : إحدا هما مجموعة اللغات السامية ؛ وثانيهما مجموعة اللغات الحامية. وتشتمل المجموعة الأولى على طائفتين :

ا. اللغات السامية الشمالية وتشمل اللغات الأكادية Accadien أو الأشسورية البابلية Assyro - Babyloniennes ، واللغات الكنعانية (العبرية والفينيقية). واللغات الآامية. r. اللغات السامية الجنوبية وتشمل العربية واليمنية القديمة واللغات الجبشية السامية. 
وأما مجموعة اللغات الحامية ، فتنتظم ثلاث طوائف : أ. اللغات المصرية ، وتشمل المصرية القديمة والقبطية. ب. اللغات الليبية أو البربرية ، وهي لغات السكان الأصلين.

لشمال أفريقيا (طرابلس وتونس والجزائرومر اكش والصحراء والجزر المتاخمة لها) ،

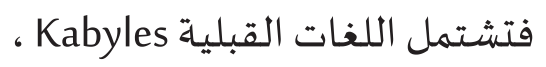

r. اللغات الكوشيتية Couchitiques وهي لغات السكان الأصلين للقسم الشرق من

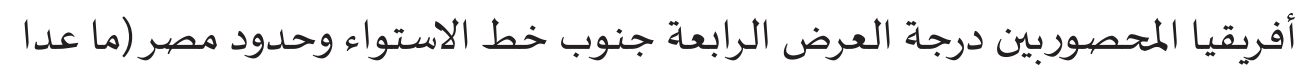

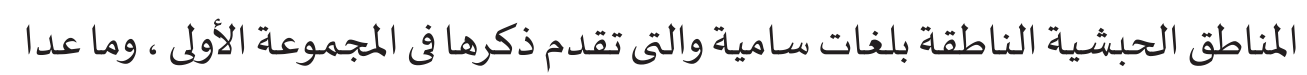

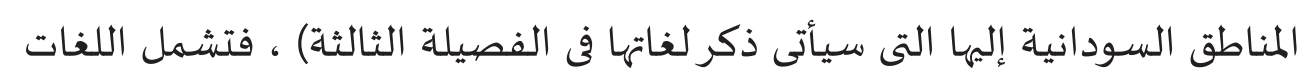

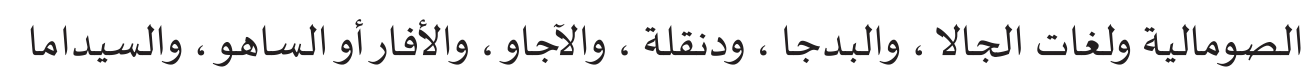
Somalie, Galla, Bedja, Dankali, Agaw, Afar, ou Saho إلخ ، بل.....

\section{الفصيلة الثالثة : اللغات الطورانية Language Taouraniennes}

أطلق مكس مولر وبونسن Bunsen اسم "اللغات الطورانية ، على طائفة من اللغات

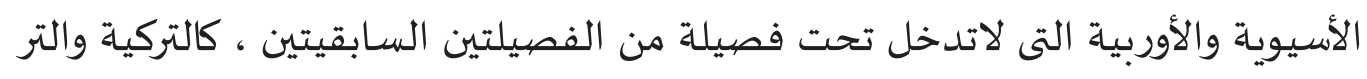

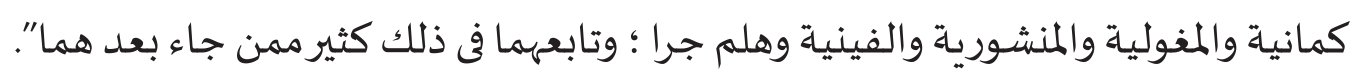
فاللغات الطورنية ليست إذن فصيلة بالمعنى الصحيح لهذه الكملة أي مجوعاة ترجع إلى إلى إدهات

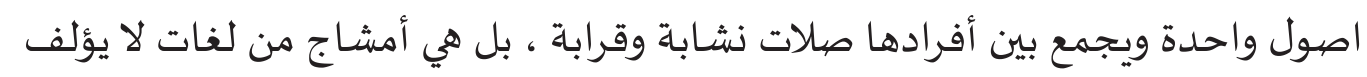
بينها إلا صففة سلبية. 
فقد نشأت اللغة العربية ، كما نشأت سائر اللغات التى عرفتها امم العالم ، من طريق الإشارة ومحاكاة اصوات الطبيعة والحيوان. ثم عملت فيها عوامل النمر والنطور حتى نضجت واكتملت كما أن اللغة العربية فرع من فصيلة كبيرة.

\section{المراجع}

طعيمة، رشدي أحمد. 1919 ـ تعليم العربيّة لغير النّاطقين بها. القاهرة: منشورات المنظة الإسلاميّة للتربية والثقافة .

طعيمة،, رشدي أحمد ومحمد السيد المناع. I . . ـ تلدريس العربية في التعلم العام، نظريات وتجارب. دار الفكر العربي .

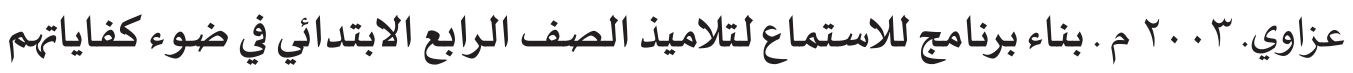

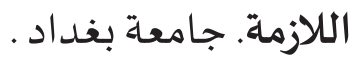

منظور, ابن, جمال الدين محمد. r199 ـ لسـان العرب. بيروت: دار احياء التراث العربي,

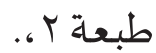

يونس, فتحي علي وعيد الرّؤوف الشيخ. ؟ . . م ـ المرجع فى تعليم اللغة العربيّة للأجانب, القاهرة: دار مكتبة و هيبة . 